\title{
Epidemiología de la Onicomicosis Pedis en adultos hospitalizados en un servicio de medicina en un hospital de tercer nivel en Chile
}

\section{Epidemiology of Onychomycosis pedisin hospitalized adults at the internal medicine ward of a high complexity hospital in Chile}

\author{
Gubelin H. ${ }^{1 *}$, Hasbún P. ${ }^{1}$, Silva C. ${ }^{2}$, Guglielmetti A. ${ }^{3}$, Espinoza A. ${ }^{4}$ \\ ${ }^{1}$ Residente de Dermatología. Universidad de Los Andes, Santiago de Chile. \\ ${ }^{2}$ Residente de Dermatología. Universidad del Desarrollo, Santiago de Chile. \\ ${ }^{3}$ Dermatólogo. Jefe de cátedra de Dermatología, Universidad de Valparaíso, Chile. \\ ${ }^{4}$ Patólogo. Director de Laboratorio Citolab, Santiago de Chile. \\ *Autor para correspondencia: hans.gubelin@ug.uchile.cl
}

RECIBIDO: 20 de abril 2021

APROBADO: 24 de julio 2021

DOI: $10.22370 /$ bolmicol.2021.36.1.2904

Palabras claves: Onicomicosis, Onicopatías, Microscopía, Hidróxido de potasio: Técnica de Schiff. Keywords: Onychomycosis; Nail disease; Microscopy; Potassium hydroxide; Periodic acid-schiff reaction.

\section{RESUMEN}

La prevalencia global de la onicomicosis pedis es de $4,3 \%$, y en hospitalizados puede llegar hasta $8,9 \%$. Aun así, se propone que está ampliamente subdiagnosticada. Personas añosas con comorbilidades presentan mayor riesgo de onicomicosis pedis y de sus complicaciones. Se examinaron aleatoriamente a 64 pacientes hospitalizados en el Servicio de Medicina del Hospital San José. A aquellos con signos clínicos de onicomicosis pedis se les realizó un examen micológico directo (MD) y estudio histopatológico de un corte de uña teñido con PAS (Bp/PAS). Muestra de 64 pacientes, un $78,1 \%$ presentó onicomicosis pedis clínica y en un 70,3\% se confirmó el diagnóstico con MD y/o
Bp/PAS positivo. De los pacientes con onicomicosis confirmada, el promedio de edad fue de 67,8 +/- 12,3 años. Un 44\% correspondió al sexo femenino y un $56 \%$ al sexo masculino. La onicomicosis pedis en el servicio de medicina interna del Hospital San José es una condición frecuente. El conjunto de MD y Bp/PAS podría ser considerado como una buena alternativa diagnóstica.

\begin{abstract}
Onychomycosis of the toenails has a global prevalence of $4,3 \%$ and can reach up to $8,9 \%$ in hospitalized patients. It has been hypothesized that it is widely under diagnosed. Aged patients with multiple diseases have an increased risk of
\end{abstract}


Epidemiología de la Onicomicosis Pedis en adultos hospitalizados en un servicio de medicina - Gubelin H. et al.

Onychomycosis and its complications. 64 patients of the internal medicine ward were randomly selected. Those who had clinical signs of onychomycosis of the toenails were tested with direct microscopy and histological study of the nail plate with PAS staining. Of the 64 patients, $78,1 \%$ (50) had clinical signs of onychomycosis of the toenails and in 70,3\% (45) the diagnosis was confirmed either by direct microscopy and/or by histological study of the nail plate with PAS staining. The mean age for the group with onychomycosis was $67,8+/-12,3$ ages. $44 \%$ were female and $56 \%$ were male. Onychomycosis of the toenails is a frequent condition at the internal medicine ward of the San José Hospital. The direct microscopy together with the histological study of the nail plate with PAS staining seem to be a good diagnosis alternative.

\section{INTRODUCCIÓN}

La onicomicosis es una infección de la lámina ungueal producida por hongos, que afecta principalmente a las uñas de los pies y da cuenta de hasta un $50 \%$ de las onicopatías. ${ }^{(1,2)} \mathrm{La}$ onicomicosis pedis es una enfermedad cuya prevalencia se encuentra en aumento. ${ }^{(3)}$ Estudios epidemiológicos estiman una prevalencia global de onicomicosis de los pies de 4,3\%, mientras que en población hospitalizada alcanza un $8,9 \%$. ${ }^{(2,3)}$ Aún así, algunos autores proponen que es una enfermedad subdiagnosticada, por lo que es posible que la prevalencia sea aún mayor. (4) Entre los factores de riesgo para onicomicosis pedis, se incluyen: tinea pedis, trauma ungueal, diabetes, enfermedad vascular periférica, inmunosupresión, edad mayor de 60 años, entre otros. ${ }^{(2,3)}$ En cuanto a los agentes etiológicos, en el mundo, los dermatofitos son los más frecuentes con un $63 \%$ de los casos. Trichophyton rubrum es el más prevalente con hasta un $91.3 \%$ de los pacientes. Entre un 13$20 \%$ corresponde a no dermatofitos y $10-20 \%$ a levaduras, dentro de ellas la más frecuente Candida albicans. ${ }^{(2-3)}$
La sospecha diagnóstica se basa en la historia clínica, el examen físico y la dermatoscopía. ${ }^{(3)}$ Se recomienda la confirmación diagnóstica por laboratorio, debido a que múltiples condiciones imitan la presentación clínica de la onicomicosis. ${ }^{(5)}$. El gold standard para el diagnóstico, sigue siendo el examen micológico directo (MD) más el cultivo micológico (CM), aunque se ha sugerido que el estudio histopatológico de un corte de uña teñido con PAS (Bp/PAS) es el examen de mejor sensibilidad diagnóstica. (1) En un estudio realizado en Chile, se observó que la sensibilidad del MD + Bp/PAS alcanzaba hasta un 97,9\%. ${ }^{(6)}$

La onicomicosis pedis es reservorio para recidiva de infecciones cutáneas frecuentes como tinea pedis, cruris o corporis. Personas con ciertas enfermedades como inmunosupresión o diabetes, presentan mayor riesgo de desarrollar micosis cutánea, la cual predispone a infecciones bacterianas como celulitis y erisipela. (7) Además, la onicomicosis repercute sobre la salud psicológica y calidad de vida de las personas. ${ }^{(8)}$

En Chile, no se han reportado estudios epidemiológicos de onicomicosis pedis en pacientes hospitalizados por causas médicas. Esta población es de particular interés por sus factores de riesgo, en quienes la onicomicosis podría ser la puerta de entrada a otras infecciones. Por esta razón, el objetivo del presente trabajo fue evaluar la situación epidemiológica de onicomicosis pedis en una población de pacientes hospitalizados en el servicio de medicina del Hospital San José, Santiago de Chile.

\section{MATERIALES Y MÉTODOS}

Este estudio cuenta con la aprobación del Comité de Ética Metropolitano Norte. 
Epidemiología de la Onicomicosis Pedis en adultos hospitalizados en un servicio de medicina - Gubelin H. et al.

$\underline{\text { Selección de pacientes }}$

Se examinaron aleatoriamente a 64 pacientes hospitalizados en el Servicio de Medicina del Hospital San José. A todos los pacientes se les solicitó consentimiento informado. Ante la sospecha de onicomicosis, se obtuvieron fotografías estandarizadas, se definió el subtipo clínico y se tomaron las muestras de la lámina ungueal preferentemente afectada. Para la clasificación clínica de los pacientes que presentaban signos de onicomicosis se utilizó la clasificación propuesta por Hay y col. (2011), publicada en la Journal of the American Academy of Dermatology.

\section{Obtención de la muestra}

Se obtuvieron 2 muestras de lámina ungueal en cada paciente con sospecha clínica de onicomicosis pedis. La primera para su análisis histopatológico (Bp/PAS) y la segunda para MD y CM.

Para el MD, cada muestra se tomó haciendo un raspado de la zona más afectada de la uña, utilizando un bisturí №20, obteniendo un material escamoso de fácil desintegración, que fue depositado en frascos de plástico estériles con tapa rosca, rotulados con los datos de cada paciente.

Para la Bp/PAS, cada muestra se obtuvo de un corte de la uña afectada, con un alicate frontal podológico estéril, siendo almacenadas en formalina (relación 3:1) dentro de frascos estériles, rotulados con los datos de cada paciente.

Las muestras que se tomaron para $\mathrm{CM}$ fueron expuestas involuntariamente a vapores de formalina. Por lo tanto, no se realizó CM por considerar su rendimiento poco confiable.

Técnica del Micológico directo (MD)

Cada muestra obtenida para este estudio fue extendida sobre un portaobjeto y sometida a solución de hidróxido de potasio $10 \%(\mathrm{KOH}$ 10\%). Una vez transcurridos 20 minutos, se evaluó la presencia de elementos fúngicos a la microscopía óptica.

Técnica estudio histopatológico de un corte de uña teñido con PAS (Bp/PAS)

Cada muestra obtenida para este estudio se incluyó en parafina con el fin de obtener un corte mediante micrótomo. La laminilla de tejido incluida se extendió en un portaobjetos y posteriormente se le realizaron tinciones de PAS (ácido peryódico de schiff) y Grocott, especiales para hongos. Se evalúo la presencia de elementos fúngicos bajo microscopía óptica.

Análisis de los datos

El procesamiento de los datos, el análisis descriptivo y comparativo, y el análisis de fiabilidad se realizó con el programa estadístico STATA 13®.

\section{RESULTADOS}

En total, 64 pacientes fueron examinados para la búsqueda de signos clínicos de onicomicosis pedis. El promedio de edad fue de $65,5+/-14,3$ años con rango entre 26 y 90 años, y el 53,1\% correspondió al sexo femenino. El 78,1\% (50) de los pacientes examinados presentó signos clínicos de onicomicosis pedis y el 70,3\% onicomicosis confirmada con MD y/o Bp/PAS. De los pacientes con onicomicosis confirmada, el promedio de edad fue de 67,8 +/- 12,3 años con rango entre 26 y 90 años, y el $56 \%$ correspondió al sexo masculino (Tabla 1).

En cuanto a los pacientes con signos clínicos de onicomicosis (50), el diagnóstico se confirmó en el $60 \%$ de los casos con MD y Bp/PAS, en el $26 \%$ solo con MD y en el $4 \%$ solo con Bp/PAS. Un $10 \%$ resultó negativo para ambos exámenes (Tabla 2). Respecto a los casos confirmados (45), el MD resultó positivo en el 95,6\% mientras que la Bp/PAS en el 71,1\%.

Con respecto a los resultados obtenidos mediante MD, en $48,9 \%$ se observaron micelios, 
Epidemiología de la Onicomicosis Pedis en adultos hospitalizados en un servicio de medicina - Gubelin H. et al.

en $39,5 \%$ micelios con artroconidios, en $9,3 \%$ levaduras y en $2,3 \%$ microconidios (Tabla 2).

Dentro de los subtipos clínicos de onicomicosis, la distrofia total fue las más frecuente, con un
$51,1 \%(23 / 45)$ de los casos (Figura 1), seguido por la subungueal distal y lateral en el $44,4 \%$ (20/45) de los casos (Figura 2). Adicionalmente, hubo un caso de dermatofitoma lineal (Figura 3) y con un patrón mixto.

Tabla 1: Caracterización epidemiológica de los pacientes

\begin{tabular}{ccc}
\hline & Total de la muestra & Onicomicosis $(+) *$ \\
\hline Sexo & $\%(\mathbf{n})$ & $\%(\mathbf{n})$ \\
Masculino & $46,9 \%(30)$ & $\mathbf{6 0 , 0 \%}(27)$ \\
Femenino & $53,1 \%(34)$ & $\mathbf{4 0 , 0 \% ( 1 8 )}$ \\
Total & $100 \%(64)$ & $100 \%(45)$ \\
Edad (años) & 65,5 & 67,8 \\
Promedio & 68 & 69 \\
Mediana & $+/-14,3$ & $+/-12,3$ \\
Desviación standard (DS) & $26-90$ & $29-90$ \\
Rango & & \\
\hline
\end{tabular}

*Se consideraron a los pacientes con micológico directo y/o Bp/PAS positivo.

Tabla 2. Resultados de los exámenes diagnósticos

\begin{tabular}{|c|c|c|c|}
\hline \multirow{2}{*}{ Examen } & \multicolumn{2}{|c|}{ Resultado } & \multirow{2}{*}{ Total } \\
\hline & Positivo \% (n) & Negativo \% (n) & \\
\hline Onicomicosis clínica & $78,1 \%(50)$ & $21,9 \%(14) *$ & 64 \\
\hline Onicomicosis confirmada $* *$ & $90,0 \%(45)$ & $10 \%(5)$ & 45 \\
\hline Micológico directo & $86,0 \%(43)$ & $14,0 \%(7)$ & $50 *$ \\
\hline Micelio & $48,8 \%(21)$ & - & - \\
\hline Micelio con artroconidios & $39,5 \%(17)$ & - & - \\
\hline Levaduras & $9,3 \%(4)$ & - & - \\
\hline Microconidios & $2,3 \%(1)$ & - & - \\
\hline Bp/PAS & $64,0 \%(32)$ & $36,0 \%(18)$ & $50 *$ \\
\hline \multicolumn{4}{|c|}{$\begin{array}{l}\text { * No se realizó MD ni Bp/PAS a pacientes sin onicomicosis clínica. } \\
\text { ** Se consideró onicomicosis confírmada en todo paciente con MD o Bp/PAS positivo. }\end{array}$} \\
\hline \multicolumn{4}{|c|}{ Relación entre resultado de los exámenes } \\
\hline Onicomicosis Clínica & MD & Bp/PAS & $\%(n)$ \\
\hline+ & + & + & $60,0 \%(30)$ \\
\hline+ & + & - & $26,0 \%(13)$ \\
\hline+ & - & + & $4 \%(2)$ \\
\hline+ & - & - & $10 \%(5)$ \\
\hline \multicolumn{3}{|c|}{ Total } & $100 \%(50)$ \\
\hline
\end{tabular}


Epidemiología de la Onicomicosis Pedis en adultos hospitalizados en un servicio de medicina - Gubelin H. et al.

Tabla 3. Clasificación de las onicomicosis

\begin{tabular}{ccc}
\hline Subtipo & \% & $\mathbf{n}$ \\
\hline Distrofia total & $\mathbf{4 6 , 0 \%}$ & $\mathbf{2 3}$ \\
Subungueal distal y lateral & $\mathbf{4 0 , 0 \%}$ & $\mathbf{2 0}$ \\
Dermatofitoma & $\mathbf{2 , 0 \%}$ & 1 \\
Compromiso ungueal mixto & $\mathbf{2 , 0 \%}$ & 1 \\
\hline Total & $\mathbf{1 0 0}$ & $\mathbf{4 5}$ \\
\hline
\end{tabular}

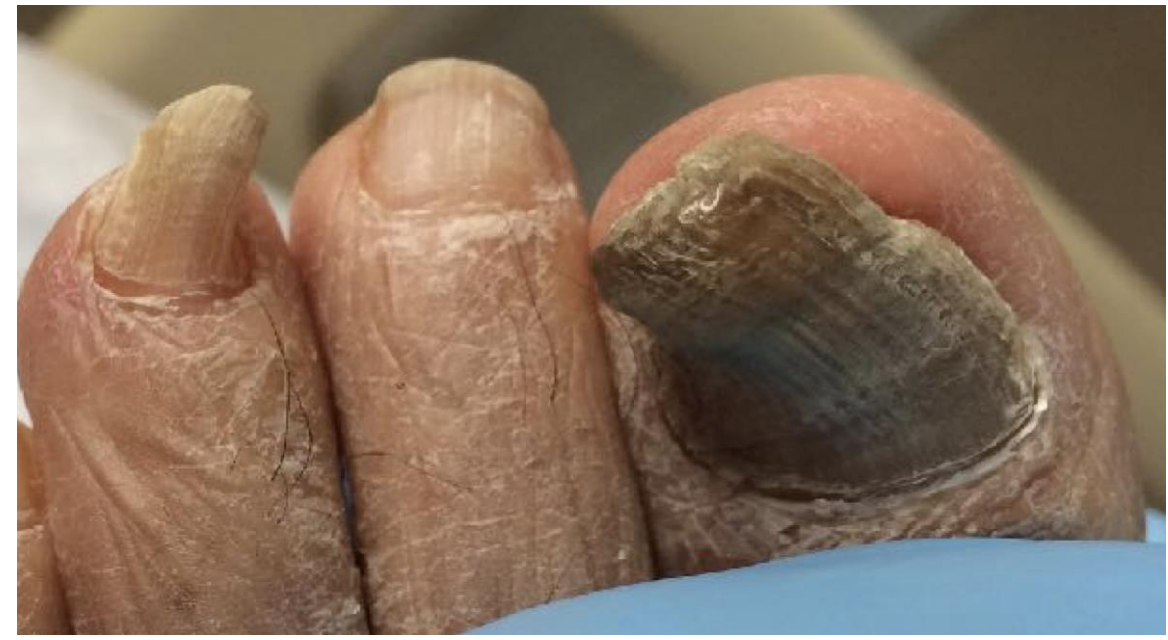

Figura 1. Onicomicosis distrofica total primer dedo del pie izquierdo

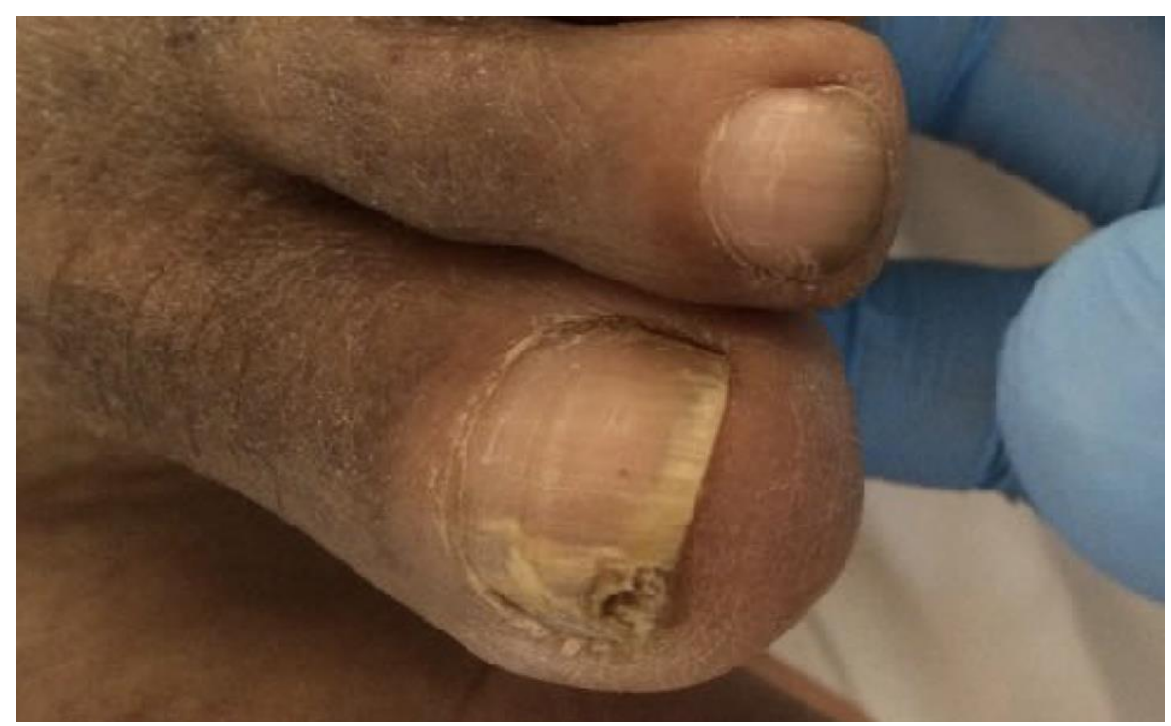

Figura 2. Onicomicosis distal lateral en primer dedo del pie izquierdo. 
Epidemiología de la Onicomicosis Pedis en adultos hospitalizados en un servicio de medicina - Gubelin H. et al.

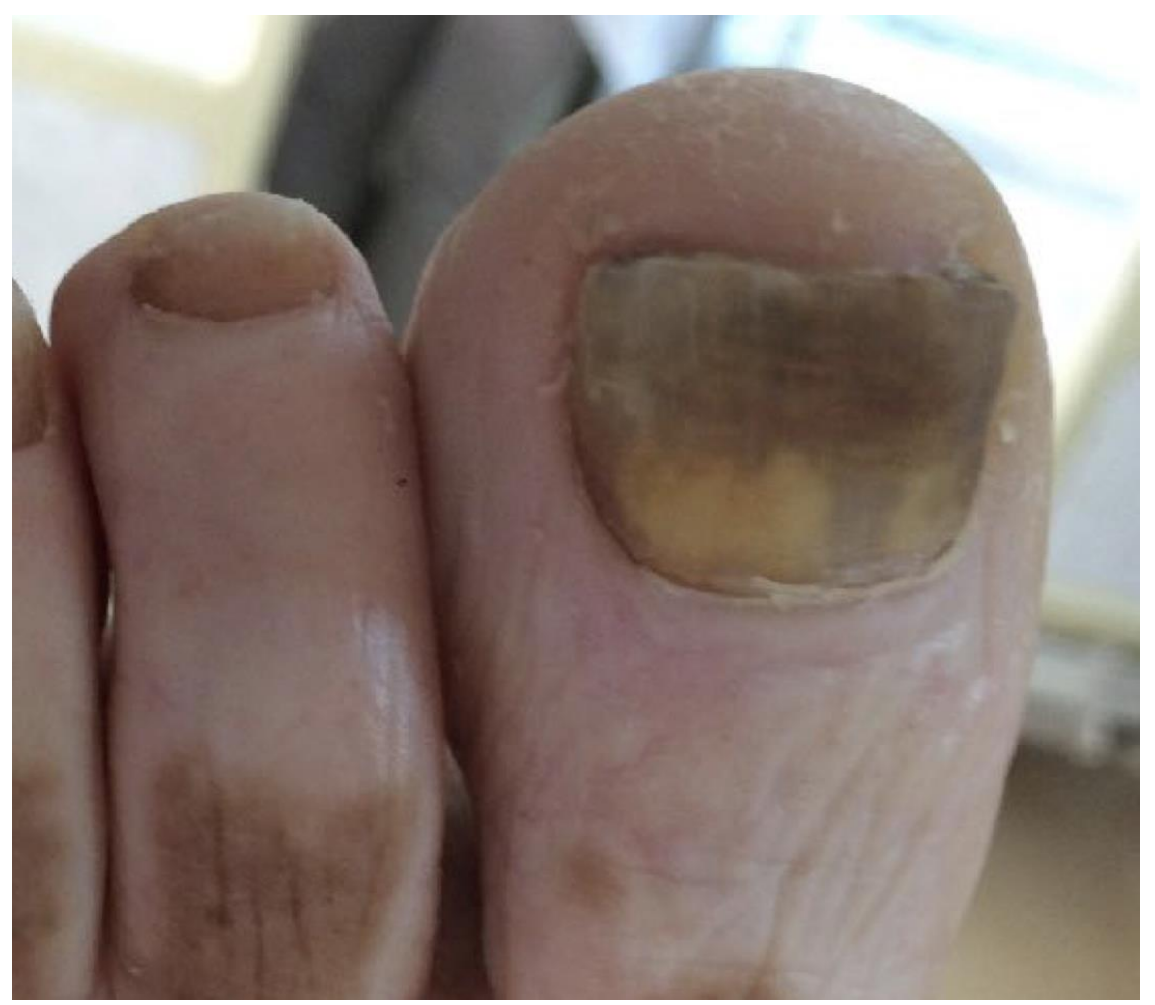

Figura 3. Onicomicosis subtipo dermatofitoma del primer dedo del pie izquierdo

\section{DISCUSIÓN}

En el presente estudio, la frecuencia de onicomicosis pedis confirmada $(70,3 \%)$ en pacientes hospitalizados supera a la descrita en la literatura. ${ }^{(2,3)}$ Estas diferencias pueden deberse a factores geográficos, puesto que cada región presenta tasas distintas de prevalencia, $\mathrm{y}$ a características propias de la población hospitalizada en servicios de medicina, tales como edad avanzada, múltiples comorbilidades y factores de riesgo para padecer esta condición.

En una gran proporción de los pacientes con sospecha clínica de onicomicosis pedis se logró la confirmación diagnóstica con MD y/o Bp/PAS. El MD constituye una de las pruebas diagnósticas más frecuentemente utilizadas ante la sospecha de onicomicosis, puesto que es simple, rápida y no requiere mayor infraestructura; sin embargo, los resultados dependerán de la experiencia del operador. En el presente estudio, el MD fue la técnica que obtuvo un mayor número de casos confirmados, lo cual probablemente tiene relación con el proceso de toma de muestra (estandarizado), una cantidad adecuada de aquella, que permitiera su análisis, y la realización de este por manos expertas, en un laboratorio de micología.

El CM es considerado el gold standard en el diagnóstico de onicomicosis, ${ }^{(9)}$ y se utiliza en conjunto con el MD en la práctica. Además de identificar la presencia de elementos fúngicos, permite determinar el agente etiológico. Sin embargo, la sensibilidad de esta prueba puede disminuir considerablemente por diversos factores que afectan la viabilidad de la muestra, tales como el uso previo de tratamientos antifúngicos (tópicos o sistémicos), una toma de muestra inadecuada, la exposición de esta a algún agente fungicida y la demora en la 
Epidemiología de la Onicomicosis Pedis en adultos hospitalizados en un servicio de medicina - Gubelin H. et al.

siembra, entre otros factores. Por otra parte, los resultados del CM en general tardan entre 3 a 4 semanas y a veces, ante la presencia aislada de hongos no dermatofitos, resulta difícil distinguirlos de un contaminante ambiental. ${ }^{(9)}$

La Bp/PAS en general se solicita ante la sospecha de onicomicosis con CM y MD negativos. Esta prueba ha mostrado una sensibilidad elevada, en algunos estudios mayor que el MD y en otros menor. ${ }^{(9,10)}$ Tiene un costo mayor que el MD, y al igual que este, no permite reconocer el agente etiológico.

Cada prueba por separado tiene una menor sensibilidad que en conjunto. En cuanto al MD y $\mathrm{Bp} / \mathrm{PAS}$, ambas técnicas tienen una sensibilidad de $48 \%$ y $53 \%$ por sí solas $^{(9)}$, sin embargo, esta mejora al usarlas en conjunto ${ }^{(6)}$, lo mismo ocurre con el MD y CM.

Respecto al subtipo clínico, la onicomicosis subungueal distal lateral es la más prevalente en la población general, mientras que en nuestro estudio fue el subtipo distrófica total, lo cual se puede deber a múltiples factores, tales como edad avanzada de los pacientes, comorbilidades y nivel socioeconómico. Dichos factores condicionan una atención tardía por una falta de oportunidad de acceso a la atención de salud, lo cual conlleva a la progresión de la micosis y muchas veces a falta de percepción de la onicomicosis como una enfermedad que requiera tratamiento. ${ }^{(12)}$

Los pacientes de los servicios de medicina interna en los hospitales públicos de nuestro país se caracterizan en general por la presencia de múltiples comorbilidades y polifarmacia. En este contexto, la onicomicosis pasa a un segundo plano. Sin embargo, el MD es un examen simple y de bajo costo, que podría realizarse en todos los pacientes con sospecha de onicomicosis pedis que se encuentren hospitalizados, de tal manera de iniciar un tratamiento y así disminuir el riesgo de complicaciones. La onicomicosis en estos pacientes tiende a ser avanzada, como se observó en nuestro estudio, lo cual facilitaría la obtención de una muestra suficiente para realizar al menos un MD.

Aunque no era el objetivo del trabajo, hubiera sido de gran interés calcular la sensibilidad, especificidad y valores predictivos de estos exámenes. Debido a que no contamos con el $\mathrm{CM}$, no fue posible.

\section{CONCLUSIONES}

La onicomicosis en el Servicio de Medicina Interna del Hospital San José es una condición frecuente y sería interesante llevar a cabo otros estudios para confirmar si estos resultados corresponden a una realidad en otros centros hospitalarios del país.

El MD y cultivo de hongos son los exámenes de elección ante la sospecha de onicomicosis, y ante un resultado negativo de estos últimos, la $\mathrm{Bp} / \mathrm{PAS}$ resulta de utilidad.

La onicomicosis es una enfermedad prevalente, por lo tanto, siendo una patología de simple sospecha, diagnóstico y manejo, debiese ser una entidad para considerar durante una hospitalización. Dado que el MD es un examen económico y fácil de realizar, es recomendable que sea tomado durante su estadía. Además, se debería educar al paciente para consultar por tratamiento de su patología de forma ambulatoria una vez dado de alta.

\section{AGRADECIMIENTOS}

Universidad de Valparaíso, Citolab, Skinmed. Dr. Gonzalo Hevia y Dr. Juan Pablo Del Río.

Conflicto de interés: los autores no tienen conflictos de interés. 
Epidemiología de la Onicomicosis Pedis en adultos hospitalizados en un servicio de medicina - Gubelin H. et al.

\section{REFERENCIAS}

1. Wilsmann-Theis D, Sareika F, Bieber T, Schmid-Wendtner M-H, Wenzel J. New reasons for histopathological nail-clipping examination in the diagnosis of onychomycosis. J Eur Acad Dermatol Venereol. 2011; 25 (2): 235-7.

2. Sigurgeirsson B, Baran R. The prevalence of onychomycosis in the global population: a literature study. J Eur Acad Dermatol Venereol. 2014; 28 (11): 1480-91.

3. Gupta AK, Versteeg SG, Shear NH. Onychomycosis in the 21st Century: An Update on Diagnosis, Epidemiology, and Treatment. J Cutan Med Surg. 2017; 21 (6): 525-39.

4. Villanueva J, Díaz C, Luna J. Perfil epidemiológico de la onicomicosis en un servicio de dermatología de un hospital de tercer nivel en Cali, Colombia. Rev Asoc Colomb Dermatol. 2013; 21 (1): 31-37.

5. Ameen M, Lear JT, Madan V, Mohd Mustapa MF, Richardson M. British Association of Dermatologists' guidelines for the management of onychomycosis 2014. Br J Dermatol. 2014; 171 (5): 937-58.

6. Gatica J, Arceu M, Muñoz L, Espinoza M, Sazunic I, Honeyman J, et al. Onicomicosis: comparación de trés métodos disgnósticos en pacientes del archipiélago de Juan Fernández. PIEL. 2017; 32 (3): 126-31.

7. Bristow IR, Spruce MC. Fungal foot infection, cellulitis and diabetes: a review. Diabet Med. 2009; 26 (5): 548.

8. Gupta, A, Mays, R. The Impact of Onychomycosis on Quality of Life: A Systematic Review of the Available Literature. Skin appendage Disord. 2018; 4 (4): 208-216.

9. Gupta AK, Mays RR, Versteeg SG, Shear NH, Piguet V. Update on current approaches to diagnosis and treatment of onychomycosis. Expert Rev Anti Infect Ther. 2018; 16 (12): 929-938.

10. Lecerf P, Abdy S, Vollono L, Pastushenko I, Richert B, André J. Direct examination, histopathology and fungal culture for the diagnosis of onychomycosis: A retrospective, comparative study on 2245 specimens. Mycoses. 2021; 64 (2): 187-193.

11. Hay RJ, Baran R. Onychomycosis: a proposed revision of the clinical classification. $\mathrm{J}$ Am Acad Dermatol. 2011; 65 (6): 1219-27.

12. Seebacher C, Brasch J, Abeck D, Cornely O, Effendy I, Ginter-Hanselmayer G, et al. Onychomycosis. J Dtsch Dermatol Ges J Ger Soc Dermatol. 2007; 5 (1): 61-6. 